Internat. J. Math. \& Math. Sci.

Vol. 23, No. 3 (2000) 211-216

S0161171200001563

(C) Hindawi Publishing Corp.

\title{
FREE MINIMAL RESOLUTIONS AND THE BETTI NUMBERS OF THE SUSPENSION OF AN $n$-GON
}

\author{
TILAK DE ALWIS
}

(Received 31 July 1998)

\begin{abstract}
Consider the general $n$-gon with vertices at the points $1,2, \ldots, n$. Then its suspension involves two more vertices, say at $n+1$ and $n+2$. Let $R$ be the polynomial ring $k\left[x_{1}, x_{2}, \ldots, x_{n}\right]$, where $k$ is any field. Then we can associate an ideal $I$ to our suspension in the Stanley-Reisner sense. In this paper, we find a free minimal resolution and the Betti numbers of the $R$-module $R / I$.
\end{abstract}

Keywords and phrases. Suspension, finite abstract simplicial complex, Stanley-Reisner ideal, free-minimal resolution, Betti numbers, double complex.

2000 Mathematics Subject Classification. Primary 13H10.

1. Introduction. Consider the suspension of the $n$-gon whose vertices are at the points $1,2, \ldots, n$ (see [6]). This introduces two new vertices, say $n+1$ and $n+2$. The finite abstract simplicial complex $\Omega$ corresponding to this suspension is given by

$$
\begin{aligned}
& \Omega=\{\varnothing,\{1\},\{2\}, \ldots,\{n\},\{n+1\},\{n+2\},\{1,2\},\{2,3\}, \ldots,\{n, 1\}, \\
&\{1, n+1\},\{2, n+1\}, \ldots,\{n-1, n+1\},\{n, n+1\},\{1, n+2\}, \\
&\{2, n+2\}, \ldots,\{n-1, n+2\},\{n, n+2\}\} .
\end{aligned}
$$

Let $k$ be any field and $R=k\left[x_{1}, \ldots, x_{n+2}\right]$. By definition, the Stanley-Reisner ideal associated to $\Omega$ is given by $I=$ The ideal in $R$ generated by all the monomials of the form $x_{i_{1}} x_{i_{2}} \cdots x_{i_{r}}$, where $1 \leq i_{1}<i_{2}<\cdots<i_{r} \leq n+2$ and $\left\{i_{1}, \ldots, i_{r}\right\} \notin \Omega$ (see [3, 7]). Then, it follows that $I=\left(x_{1} x_{3}, x_{1} x_{4}, \ldots, x_{1} x_{n-1}, x_{2} x_{4}, \ldots, x_{2} x_{n}, \ldots, x_{n-2} x_{n}, x_{n+1} x_{n+2}\right)$ for $n>3$, and $I=\left(x_{1} x_{2} x_{3}, x_{4} x_{5}\right)$ for $n=3$. In the literature, the ring $R / I$ is also known as the face ring or the Stanley-Reisner ring of the finite abstract simplicial complex $\Omega$ (see $[3,7])$.

By definition, a free-minimal resolution of the $R$-module $R / I$ is an exact sequence of the form

$$
\cdots M_{i} \rightarrow \cdots \rightarrow M_{1} \rightarrow M_{0} \rightarrow \frac{R}{I} \rightarrow 0
$$

where each $M_{i}$ is a free $R$-module with the smallest possible rank. For material on free-minimal resolutions, the reader can refer to [5] or [7]. The Betti numbers $B_{i}(n)$ of the $R$-module $R / I$ are just the ranks of those free modules $M_{i}$, i.e., $B_{i}(n)=\operatorname{rank}_{R}\left(M_{i}\right)$ for $i=0,1, \ldots$. 
In this paper, we find a free-minimal resolution and the Betti numbers of the $R$ module $R / I$. Sometimes we simply refer to them as a free-minimal resolution and the Betti numbers of the suspension of the $n$-gon.

2. Some useful results. In this section, we recall some results on free-minimal resolutions and the Betti numbers of the $n$-gon. These results are needed to obtain the theorems on the suspension of the $n$-gon. The proofs of most of these theorems can be found in [1] or [2].

(1) Let $\Delta$ be the finite abstract simplicial complex corresponding to the $n$-gon with vertices at the points $1,2, \ldots, n$. Let $S=k\left[x_{1}, \ldots, x_{n}\right]$ and $J_{1}$ be the Stanley-Reisner ideal associated to $\Delta$. Then, it easily follows that $J_{1}=\left(x_{1} x_{3}, x_{1} x_{4}, \ldots, x_{2} x_{4}, \ldots, x_{2} x_{n}, \ldots\right.$, $\left.x_{n-2} x_{n}\right)$ for $n>3$, and $J_{1}=\left(x_{1} x_{2} x_{3}\right)$ for $n=3$.

(2) Let $\beta_{i}(n)$ denote the $i$ th Betti number of the $S$-module $S / J_{1}$. In other words, it is the $i$ th Betti number of the $n$-gon. Then, for $n \geq 3$,

$$
\beta_{i}(n)=\left\{\begin{array}{l}
1, \quad i=0 \\
\left(\begin{array}{c}
n \\
i+1
\end{array}\right) \frac{i(n-i-2)}{n-1}, \quad i=1,2, \ldots, n-3, \\
1, \quad i=n-2, \\
0, \quad \text { otherwise. }
\end{array}\right.
$$

(3) We can show that,

$$
0 \longrightarrow S^{\beta_{n-2}} \stackrel{f_{n-2}}{\longrightarrow} S^{\beta_{n-3}} \longrightarrow \cdots \longrightarrow S^{\beta_{1}} \stackrel{f_{1}}{\longrightarrow} S^{\beta_{0}} \stackrel{f_{0}}{\longrightarrow} \frac{S}{J_{1}} \longrightarrow 0
$$

is a free-minimal resolution of the $S$-module $S / J_{1}$. Even though we do not need the specific definitions of the maps $f_{j}$ for what follows, the inquisitive reader can find them in [1].

3. Main results. Let $J_{1}$ be the ideal in the polynomial ring $S=k\left[x_{1}, \ldots, x_{n}\right]$ as in Section 2 . Let $J$ be the ideal in the polynomial ring $R=k\left[x_{1}, \ldots, x_{n}, x_{n+1}, x_{n+2}\right]$ generated by the same generators as that of $J_{1}$.

Tensor the exact sequence (2.2) with the $k$-module $k\left[x_{n+1}, x_{n+2}\right]$, which is a free module. Hence we obtain the following exact sequence of $R$-modules.

$$
0 \longrightarrow R^{\beta_{n-2}} \stackrel{d_{n-2}}{\longrightarrow} R^{\beta_{n-3}} \longrightarrow \cdots \rightarrow R^{\beta_{1}} \stackrel{d_{1}}{\longrightarrow} R^{\beta_{0}} \stackrel{d_{0}}{\longrightarrow} \frac{R}{J} \longrightarrow 0
$$

where $d_{i}$ are the same as the maps $f_{i} \otimes i d$. This means that the following complex is exact at all places except at degree 0 :

$$
\begin{aligned}
& \begin{array}{llll}
n-2 & n-3 & 1 & 0
\end{array} \\
& 0 \longrightarrow R^{\beta_{n-2}} \stackrel{d_{n-2}}{\longrightarrow} R^{\beta_{n-3}} \longrightarrow \cdots \longrightarrow R^{\beta_{1}} \stackrel{d_{1}}{\longrightarrow} R^{\beta_{0}} \longrightarrow 0
\end{aligned}
$$


Consider the following diagram where the two rows are the same as the complex (3.2) and the vertical maps are multiplication by the element $y=x_{n+1} x_{n+2}$ of $R$.

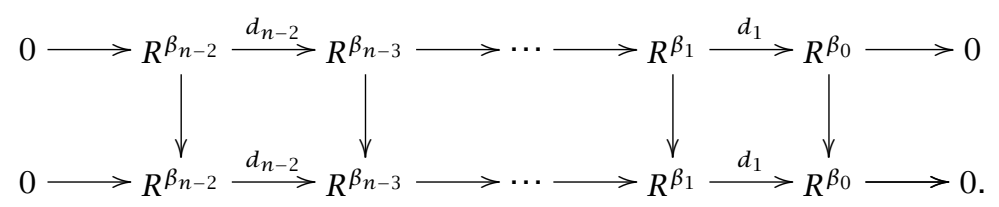

The squares in (3.3) commute, because $x_{n+1} x_{n+2}$ is an element of the ring $R$ and our maps are $R$-module homomorphisms. Hence (3.3) is a double complex, and its total complex is given by

$$
0 \longrightarrow R^{\beta_{n-2}} \stackrel{\partial_{n-1}}{\longrightarrow} R^{\beta_{n-2}} \oplus R^{\beta_{n-3}} \longrightarrow \cdots \longrightarrow R^{\beta_{1}} \oplus R^{\beta_{0}} \stackrel{\partial_{1}}{\longrightarrow} R^{\beta_{0}} \longrightarrow 0,
$$

where the differential maps $\partial_{i}: R^{\beta_{i}} \oplus R^{\beta_{i-1}} \rightarrow R^{\beta_{i-1}} \oplus R^{\beta_{i-2}}, i=1,2, \ldots, n-1$ are given by $\partial_{i}(p, q)=\left(d_{i}(p)+(-1)^{i} y q, d_{i-1}(q)\right)$ for $i=2,3, \ldots, n-2$. Obvious definitions would apply for $i=1$ and $i=n-1$. It is a routine exercise to verify that $\partial_{i-1} \circ \partial_{i}=0$.

THEOREM 3.1. The complex (3.4) is exact at all places except degree 0 at which it has homology equal to $R / I$. In other words, the following is a free resolution of $R / I$ :

$$
0 \longrightarrow R^{\beta_{n-2}} \stackrel{\partial_{n-1}}{\longrightarrow} R^{\beta_{n-2}} \oplus R^{\beta_{n-3}} \longrightarrow \cdots \rightarrow R^{\beta_{1}} \oplus R^{\beta_{0}} \stackrel{\partial_{1}}{\longrightarrow} R^{\beta_{0}} \longrightarrow \frac{R}{I} \longrightarrow 0
$$

Proof. Denote $R^{\beta_{i}}$ by $D_{i}$. Then for $i>1$, consider the sequence $D_{i+1} \oplus D_{i} \stackrel{\partial_{i+1}}{\longrightarrow}$ $D_{i} \oplus D_{i-1} \stackrel{\partial_{i}}{\longrightarrow} D_{i-1} \oplus D_{i-2}$. Suppose $(p, q) \in \operatorname{Ker} \partial_{i}$. Then $\partial_{i}(p, q)=\left(d_{i}(p)+(-1)^{i} y q\right.$, $\left.d_{i-1}(q)\right)=0$. Hence $d_{i}(p)+(-1)^{i} y q=0$ and $d_{i-1}(q)=0$. Therefore, $q \in \operatorname{Ker} d_{i-1}=$ $\operatorname{im} d_{i}$, so $q=d_{i}\left(q_{1}\right)$ for some $q_{1} \in D_{i}$. The equation $d_{i}(p)+(-1)^{i} y q=0$ yields $d_{i}(p+$ $\left.(-1)^{i} y q_{1}\right)=0$, which means that $p+(-1)^{i} y q_{1} \in \operatorname{Ker} d_{i}=\operatorname{im} d_{i+1}$. Therefore, $p+$ $(-1)^{i} y q_{1}=d_{i+1}\left(p_{1}\right)$ for some $p_{1} \in D_{i+1}$, which implies that $p=d_{i+1}\left(p_{1}\right)+$ $(-1)^{i+1} y q_{1}$. Hence, $\partial_{i+1}\left(p_{1}, q_{1}\right)=(p, q)$, i.e., $(p, q) \in \operatorname{im} \partial_{i+1}$. This shows that Ker $\partial_{i}=$ $\operatorname{im} \partial_{i+1}$ for $i>1$.

For $i=1$, we have $D_{2} \oplus D_{1} \stackrel{\partial_{2}}{\longrightarrow} D_{1} \oplus D_{0} \stackrel{\partial_{1}}{\longrightarrow} D_{0}$. Let $(p, q) \in \operatorname{Ker} \partial_{1}$. Therefore, $\partial_{1}(p, q)=d_{1}(p)+(-1) y q=0$. This yields $d_{1}(p)=y q \in \operatorname{im} d_{1}=\operatorname{Ker} d_{0}=J$. But $y \notin J$. Hence, even though $J$ is not a prime ideal of $R$, by considering the primary decomposition of $J$, one can easily obtain that $q \in J$. Therefore, the exact sequence (3.1) gives us $q=d_{1}\left(q_{1}^{\prime}\right)$ for some $q_{1}^{\prime} \in D_{1}$. Hence $d_{1}(p)=y q=y d_{1}\left(q_{1}^{\prime}\right)=d_{1}\left(y q_{1}^{\prime}\right)$, which implies that $p-y q_{1}^{\prime} \in \operatorname{Ker} d_{1}=\operatorname{im} d_{2}$. Therefore, $p-y q_{1}^{\prime}=d_{2}\left(p_{1}^{\prime}\right)$ for some $p_{1}^{\prime} \in D_{2}$. Hence, $p=d_{2}\left(p_{1}^{\prime}\right)+y q_{1}^{\prime}$. Now we have two equations $d_{2}\left(p_{1}^{\prime}\right)+y q_{1}^{\prime}=p$, and $d_{1}\left(q_{1}^{\prime}\right)=q$ where $\left(p_{1}^{\prime}, q_{1}^{\prime}\right) \in D_{2} \oplus D_{1}$. This yields $\partial_{2}\left(p_{1}^{\prime}, q_{1}^{\prime}\right)=(p, q)$ and hence Ker $\partial_{1}=\operatorname{im} \partial_{2}$.

Finally, for $i=0$, we have $D_{1} \oplus D_{0} \stackrel{\partial_{1}}{\longrightarrow} D_{0} \rightarrow 0$. We know that $\partial_{1}(p, q)=d_{1}(p)-y q$. However, the exact sequence (3.1) implies that $d_{1}\left(D_{1}\right)=J$ and hence im $\partial_{1}=\{j-y q \mid$ $j \in J, q \in R\}=J+(y)=I$. Therefore the homology of the complex (3.4) at the zeroth spot is equal to $R / I$.

Theorem 3.2 says more about the free resolution (3.5). 
THEOREM 3.2. The sequence (3.5) is a free-minimal resolution of the $R$-module $R / I$.

Proof. To show the minimality, it is enough to show that the maps $\partial_{i} \otimes i d$ : $\left(R^{\beta_{i}} \oplus R^{\beta_{i-1}}\right) \otimes_{R} k \rightarrow\left(R^{\beta_{i-1}} \oplus R^{\beta_{i-2}}\right) \otimes_{R} k$ are zero for $i=1,2, \ldots, n-1$ (see [4, p. 136]). However, this is an easy consequence of commutativity of the following diagram and the minimality of (2.2):

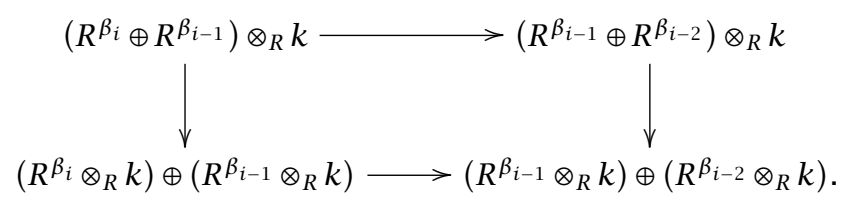

Theorem 3.3 enables us to calculate the Betti numbers $B_{i}(n)$ of the suspension of the $n$-gon.

THEOREM 3.3. Let $n \geq 3$ be a positive integer. Then the ith Betti number $B_{i}(n)$ of the suspension of the $n$-gon is given by

$$
B_{i}(n)= \begin{cases}1, & i=0, \\
\left(\begin{array}{c}
n-1 \\
2
\end{array}\right), & i=1, \\
\left(\begin{array}{c}
n \\
i
\end{array}\right) \frac{\left[n i-\left(i^{2}+i+1\right)\right]}{i+1}, & i=2,3, \ldots, n-3, \\
\left(\begin{array}{c}
n-1 \\
2
\end{array}\right), & i=n-2, \\
1, & i=n-1, \\
0, & \text { otherwise. }\end{cases}
$$

Proof. Let $n \geq 3$ be a positive integer. Since (3.5) is a free-minimal resolution, the Betti numbers of $R / I$ are just the respective ranks of the free modules appearing in (3.5). Hence, we obtain, for $n \geq 3$,

$$
B_{i}(n)= \begin{cases}\beta_{0}, & i=0, \\ \beta_{i}+\beta_{i-1}, & i=1,2, \ldots, n-2, \\ \beta_{n-2}, & i=n-1 .\end{cases}
$$

Let us denote $B_{i}(n)$ by $B_{i}$. The theorem is clear for $n=3$. Therefore assume that $n>3$. So

$$
B_{0}=\beta_{0}=\beta_{n-2}=B_{n-1}=1
$$

and

$$
B_{1}=\beta_{1}+\beta_{0}=\left(\begin{array}{l}
n \\
2
\end{array}\right) \frac{n-3}{n-1}+1=\frac{1}{2} n(n-3)+1=\frac{1}{2}(n-1)(n-2)=\left(\begin{array}{c}
n-1 \\
2
\end{array}\right)
$$


by using formula (2.1). A similar calculation shows that $B_{n-2}=\left(\begin{array}{c}n-1 \\ 2\end{array}\right)$. Now, let $1<i<$ $n-2$. The formula (2.1) again gives,

$$
\begin{aligned}
B_{i}=\beta_{i}+\beta_{i-1}= & \left(\begin{array}{c}
n \\
i+1
\end{array}\right) \frac{i(n-i-2)}{n-1}+\left(\begin{array}{c}
n \\
i
\end{array}\right) \frac{(i-1)(n-i+1-2)}{n-1} \\
= & \frac{n !}{(i+1) !(n-i-1) !} \cdot \frac{i(n-i-2)}{n-1}+\frac{n !}{i !(n-i) !} \cdot \frac{(i-1)(n-i-1)}{n-1} \\
= & \frac{n !}{(n-1)(i+1)(n-i) i !(n-i-1) !} \\
& \times[i(n-i)(n-i-2)+(i-1)(i+1)(n-i-1)] \\
= & \frac{n !}{(n-1)(i+1)(n-i) i !(n-i-1) !}\left[n^{2} i-n(i+1)^{2}+i^{2}+i+1\right] \\
= & \frac{n !}{(n-1)(i+1)(n-i) i !(n-i-1) !}(n-1)\left[n i-\left(i^{2}+i+1\right)\right] \\
= & \frac{n !}{i !(n-i) !} \frac{\left[n i-\left(i^{2}+i+1\right)\right]}{i+1}=\left(\begin{array}{c}
n \\
i
\end{array}\right) \frac{\left[n i-\left(i^{2}+i+1\right)\right]}{i+1}
\end{aligned}
$$

which proves the formula (3.7) for the case $n>3$. This completes the proof.

We can illustrate our theory with an example. For $n=4$ we get the suspension of the square, which is nothing but the familiar octahedron. Hence,

$$
\begin{aligned}
\Omega= & \{\varnothing,\{1\},\{2\},\{3\},\{4\},\{5\},\{6\},\{1,2\},\{2,3\},\{3,4\},\{4,1\}, \\
& \{1,5\},\{2,5\},\{3,5\},\{4,5\},\{1,6\},\{2,6\},\{3,6\},\{4,6\}\}, \\
R= & k\left[x_{1}, x_{2}, x_{3}, x_{4}, x_{5}, x_{6}\right], \\
I= & \left(x_{1} x_{3}, x_{2} x_{4}, x_{5} x_{6}\right) .
\end{aligned}
$$

The formula (3.7) gives us $B_{0}(4)=1, B_{1}(4)=3, B_{2}(4)=3$, and $B_{3}(4)=1$ which are the Betti numbers of the octahedron.

ACKNOWLEDGEMENT. The author wishes to thank his adviser John Eagon for all the valuable help.

\section{REFERENCES}

[1] T. de Alwis, Free minimal resolutions, Comm. Algebra 21 (1993), no. 12, 4575-4585. MR 95f:13017. Zbl 806.18009.

[2] _ Pascal type properties of Betti numbers, Internat. J. Math. Math. Sci. 17 (1994), no. 3, 545-552. MR 95c:13026. Zbl 809.13010.

[3] M. Hochster, Cohen-Macaulay rings, combinatorics, and simplicial complexes, Ring theory, II (New York), Lecture Notes in Pure and Appl. Math., vol. 26, Proc. Second Conf., Univ. Oklahoma, Dekker, 1977, pp. 171-223. MR 56 376. Zbl 351.13009.

[4] H. Matsumura, Commutative algebra, 2d ed., Mathematics Lecture Note Series, vol. 56, Benjamin/Cummings Publishing Co., Inc., Reading, M.S., 1980. MR 82i:13003. Zbl 441.13001.

[5] , Commutative ring theory, Cambridge Studies in Advanced Mathematics, vol. 8, Cambridge University Press, Cambridge, New York, 1986. MR 88h:13001. Zbl 603.13001. 
[6] J. R. Munkres, Elements of Algebraic Topology, Addison-Wesley Publishing Company, Menlo Park, Calif., 1984. MR 85m:55001. Zbl 673.55001.

[7] R. P. Stanley, Combinatorics and commutative algebra, Progress in Mathematics, vol. 41, Birkhauser Boston, Inc., Boston, M.A., 1983. MR 85b:05002. Zbl 537.13009.

De Alwis: Department of Mathematics, SOUTHEASTERn Louisiana University, HamMOND, LOUISIANA 70402, USA 


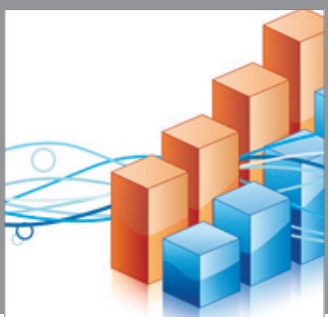

Advances in

Operations Research

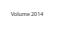

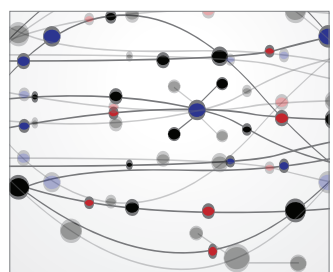

\section{The Scientific} World Journal
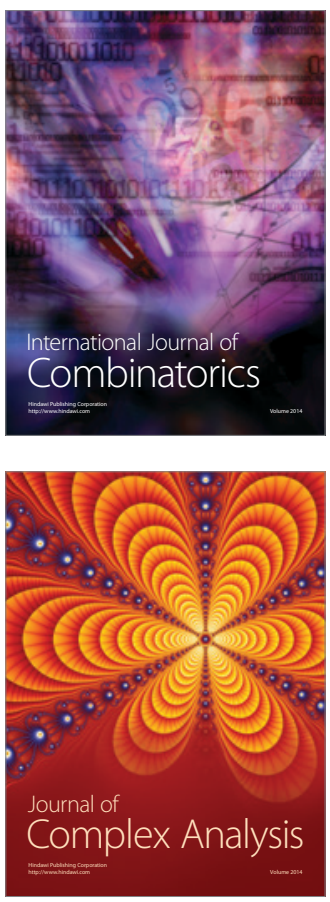

International Journal of

Mathematics and

Mathematical

Sciences
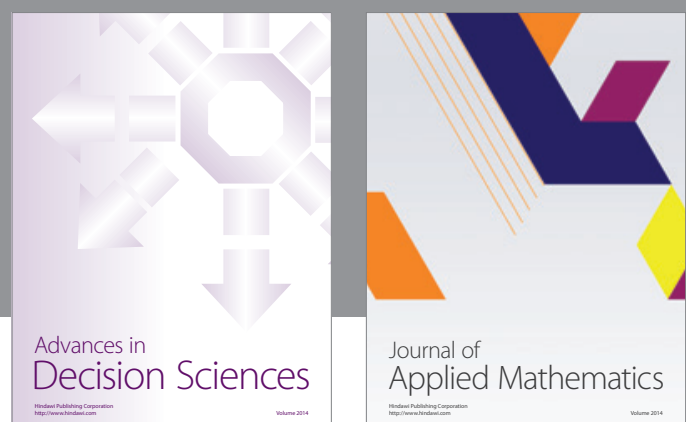

Journal of

Applied Mathematics
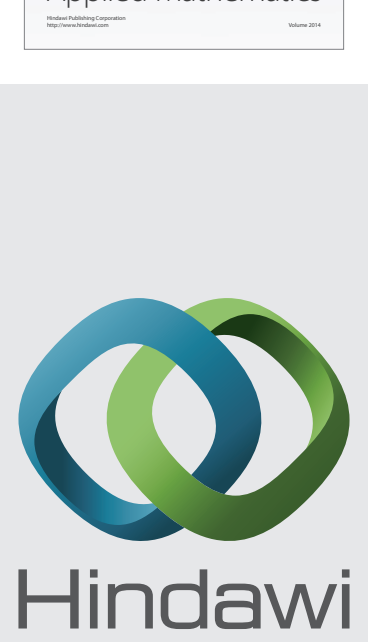

Submit your manuscripts at http://www.hindawi.com
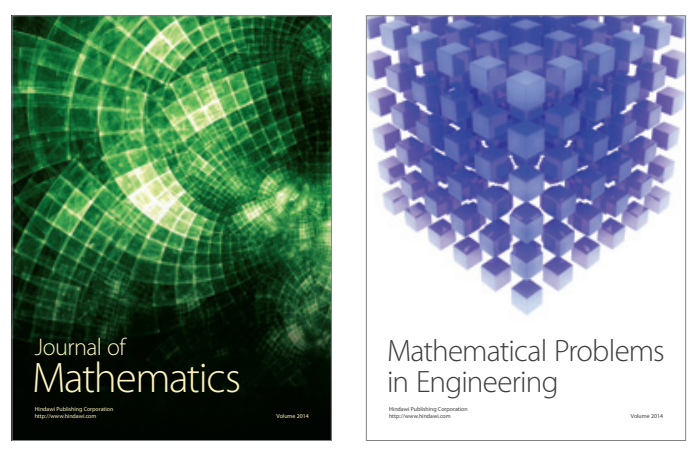

Mathematical Problems in Engineering
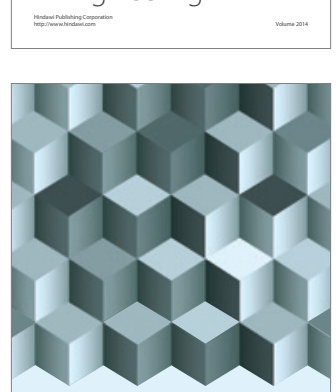

Journal of

Function Spaces
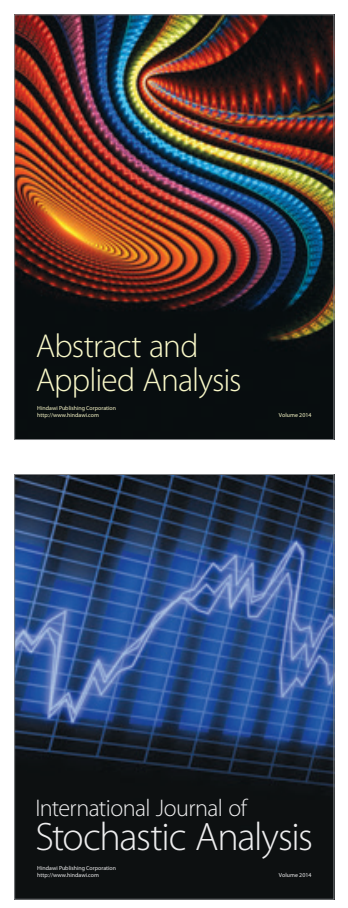

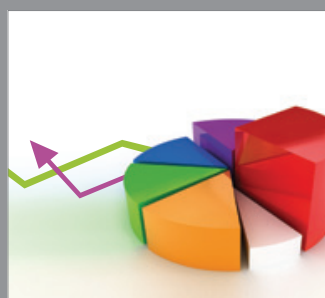

ournal of

Probability and Statistics

Promensencen
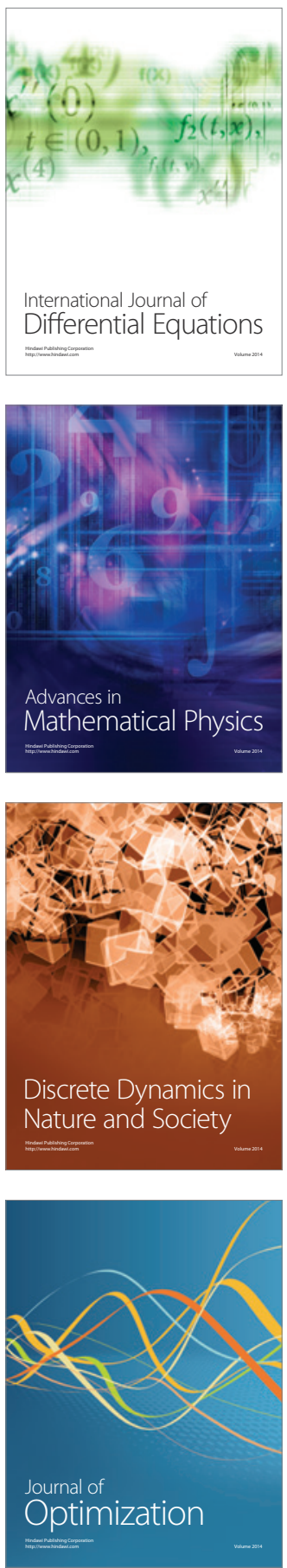